\title{
Retronasal olfactory testing using candies sent by post and for screening purposes: a feasibility study*
}

\author{
Gerold Besser', David Tianxiang Liu', Bernhard Prem', Darina labloncsik?' \\ Eleonore Pablik², Christian A. Mueller', Bertold Renner ${ }^{3,4}$
}

' Department of Otorhinolaryngology and Head and Neck Surgery, Medical University of Vienna, Austria

2 Section for Medical Statistics, CeMSIIS, Medical University of Vienna, Austria

${ }^{3}$ Institute of Experimental and Clinical Pharmacology and Toxicology, Friedrich-Alexander-Universität Erlangen-Nürnberg,

Erlangen, Germany

${ }^{4}$ Institute of Clinical Pharmacology, Medical Faculty Carl Gustav Carus, Technische Universität Dresden, Dresden, Germany
Rhinology 58: 3, 218 - 225, 2020

https://doi.org/10.4193/Rhin 19.230

*Received for publication:

July 3, 2019

Accepted: January 13, 2020

\begin{abstract}
Background: The olfactory system is able to detect external odours through the orthonasal- and internal odours through the retronasal route. Flavour perception strongly relies on the sense of smell and this back route. In contrast to orthonasal, retronasal olfactory tests (ROT) are less frequently applied, although testing should be recommended for several reasons. The aim of the present investigation was to propose a suitable form of ROT for home-testing (and postal distribution) and evaluate a retronasal screening test.
\end{abstract}

Methodology: Initially, 111 participants were tested using a 27-item version of the Candy Smell Test (CST). Fifty-four participants performed retesting, of which 25 subjects did so in a home-setting being supplied with professionally packed "candy-chains". Seven candies were chosen by means of hit rate differences in normosmics and severely hyposmics/anosmics. The 7-CST is designed in a non-forced-choice fashion with same seven flavours to choose from.

Results: For the 27-item CST both groups (subjects performing home-testing and those performing retesting at the clinic) showed highly significant test-rest-reliabilities. The 7-CST was capable of discriminating healthy from diseased subjects when being tested in 116 healthy subjects and 47 patients suffering from olfactory dysfunction.

Conclusion: The CST is suitable for home-testing and postal distribution. The new 7-item CST can be valuable for rapidly revealing anosmics. These findings help in further standardizing ROT, may encourage rhinologists to more routinely evaluate retronasal olfactory abilities and pave the way for larger epidemiologic studies also in regard to food preferences and nutritional behaviour.

Key words: anosmia, flavour, mail distribution, self-administration, taste

\section{Introduction}

The widely underestimated contribution of the sense of smell to flavour perception leads to a "Smell-Taste-Confusion" (1,2). OIfaction is able to sense external odours through the orthonasal route and internal odours through the retronasal route ${ }^{(2)}$. Nasal air outflow during expiration is essential for odorant delivery to the olfactory epithelium ${ }^{(3)}$. These retronasally perceived odours are often mislocalized to the mouth, hence interpreted as "taste", whereas taste (gustation) refers only to the basic qualities sweet, sour, salty, bitter and umami ${ }^{(4)}$. In contrast to orthonasal, retro- nasal perception solely includes food and beverage volatiles and activates many brain processing circuits, also related to gustatory function ${ }^{(5)}$. Furthermore, adaption to odors seems to be depending on route, possibly diminished for retronasally delivered odors ${ }^{(6)}$.

Unaware of these relations, patients with olfactory dysfunction frequently realize as late as when orthonasal and retronasal olfactory, as well as gustatory function are tested separately, the effects of their smell, but not taste impairment. Beside this "educational" benefit of retronasal olfactory testing, disease 
specific differences (e.g., chronic rhinosinusitis with nasal polyps, CRSwNP) in ortho- vs. retronasal olfactory performance have been reported and underline the legitimation for retronasal tests ${ }^{(7,8)}$.

Numerous different tools are available in orthonasal olfactory testing ${ }^{(9,10)}$. Short screening tests are favourable in settings with time restrictions ${ }^{(9-11)}$ and sufficient for general practitioners ${ }^{(12)}$. Very popular, the University of Pennsylvania Smell Identification Test (UPSIT) is comprised of booklets, containing microencapsulated odors releasable in a "scratch-and-sniff"-technique (13). The test can be self-administered and delivered by mail ${ }^{(14)}$. For retronasal olfactory function (ROF), several tools have been proposed in a clinical context: using powders available in groceries stores ${ }^{(15)}$, using candies ${ }^{(16)}$, applying aqueous solutions containing aromatic extracts with pipettes ${ }^{(17)}$ and recently using freeze-dried flavours ${ }^{(18)}$. To this point, no suitable presentation form and strategy have been advocated for ROF, which enable home-testing (i.e., postal distribution) and may also serve as a rapid screening method.

\section{Material and methods}

\section{General subjects}

The following investigation consists of two studies, which were approved by the ethics committee of the Medical University of Vienna (EK No. 1160/2018 and 1582/2018) and were conducted according to the guidelines of the Declaration of Helsinki on Biomedical Research Involving Human Subjects. All subjects $(n=287)$ provided written informed consent prior to participation and tests took place between April 2018 and May 2019. Healthy participants were recruited using invitational notices that were displayed at the university campus, whereas patients with olfactory dysfunction (OD) were recruited through our smell and taste clinic. In this population $(n=90), O D$ was most frequently due to an upper respiratory infection (36 patients, $33 \%$ ), followed by idiopathic causes (29 patients, 26.6\%) and traumatic head injuries (14 patients, 12.8\%). In four cases (3.7\%) OD was of sinunasal, in three (2.8\%) of congenital, in three (2.8\%) of neurodegenerative and in one $(0.9 \%)$ of noxious cause. Additionally, 29 patients were tested prior to rhinologic surgeries (sinus surgery and/or septorhinoplasty).

\section{Olfactory tests}

For orthonasal olfactory testing we examined odor threshold (T), odor discrimination (D) and odor identification (I) using Sniffin' Sticks (reusable odorant 'pens'; Burghart GmbH, Wedel, Germany). The testing procedures are described in detail elsewhere ${ }^{(19,20)}$. For TDI Sniffin' Sticks testing large normative data sets are available ${ }^{(21,22)}$ and anosmia can be seen at a TDI score of 16 or less and the cut-off for normosmia is equal or above $30.75^{\text {(22). }}$ For retronasal olfactory testing candies we applied a 27-item version of the Candy Smell Test (27-CST). This test was validated in a 23 -item version ${ }^{(16)}$, but recently also used in the following version ${ }^{(23)}$. In agreement with the developing group, less identifiable aromas of previous investigations ${ }^{(16,24)}$ were removed (three aromas: passion fruit, strawberry and kiwi) and others introduced (seven aromas: apricot, rhubarb, coconut, eisbonbon, plum, condensed milk and nut nougat). The candies have a diameter of approximately nine millimetre each and contain $500 \mathrm{mg}$ sorbitol and the target aroma. They were professionally manufactured at the Division of Pharmaceutics at the FriedrichAlexander University Erlangen-Nürnberg (Germany) with aromas obtained from Frey\&Lau GmbH Henstedt-Ulzburg (Germany). All aromas (i.e., candies) are of food-grade-quality.

With the sorbitol as the candy matrix, they are applicable in diabetes and do not increase the risk for dental caries, but should not be used in fructosaemia. Excessive consumption may cause diarrhoea ${ }^{(16)}$. One from four possible answers (without visual cues) had to be chosen in a forced-choice manner with a maximal attainable test score of 27 . Subjects were asked to suck or chew the candy, spit it out right after recognition (in order not to prolong testing duration) and rinse the mouth with water after each candy.

For home-testing, candies were professionally (hygienically and partly automatically) packed at a local pharmacy. Each candy was sealed in one little plastic bag and numbered. Bags were supplied with perforation lines for easy opening (no scissors needed) and attached to each other, forming a chain and enabling roll up and compression (Figure 1). One candy-chain easily fits in a standard envelope for postal distribution, including simple instructions (e.g., where to open, where to separate bags) and a smaller stamped return envelope. The CST is not yet commercially available. However, an optimized version of the CST will be developed for commercial use in the near future.

\section{Study I}

The aims of study I were to investigate identification rates of presented 27 aromas, to test for reproducibility of previous findings (16,24) and to explore the feasibility of home self-testing.

Data on 49 healthy subjects, 27 women and 22 men, mean age $31.1 \pm 10.4$ years and 62 subjects scoring below the normative range of normosmia, 34 women and 28 men, mean age $52.1 \pm 18.3$ years, was evaluated. All subjects were orthonasally tested for T, D and I and were tested retronasally with the 27-CST. Overall 54 participants performed retesting, of which 25 subjects (18 OD patients and 7 healthy subjects) were supplied with candy-chains (see above) and self-tested ROF in an unmonitored home-setting.

\section{Study II}

The aim of study II was to establish a short retronasal test in terms of a screening test by building up on data from study I. This study included 116 healthy subjects, 79 women and 37 


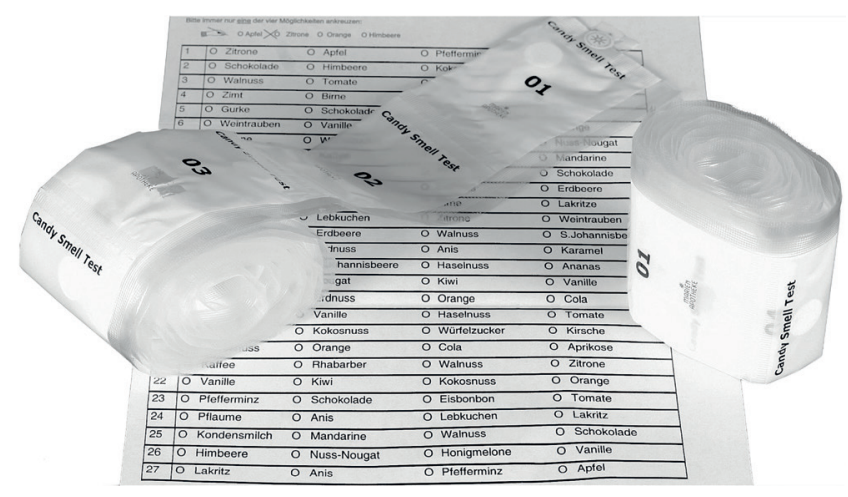

Figure 1. Professionally packed candies for home-self-testing of retronasal olfactory function. Each candy is sealed in one little plastic bag and numbered. All 27 bags form a chain and can be rolled up and compressed. One candy-chain easily fits in standard envelopes for postal distribution, including simple instructions, an answer sheet and a smaller stamped return envelope.

men, mean age 30.0 12.7 years and 47 patients with OD, 28 women and 19 men, mean age $55.9 \pm 15.0$ years. Additionally, 13 children/young adolescents, eight girls and five boys, with a mean age of 12.0 \pm 2.8 (range 7-16 years) were included. OD patients were tested for T, D and I, whereas healthy subjects were screened for OD using the 16-item identification test kit. In case of children 14-items of the identification battery (Sniffin` kids test $\left.{ }^{(25)}\right)$ were used.

Data from study I was used and analysed for hits on aromas in subjects performing above the normative range of normosmia in comparison to those scoring at TDI 20 or below in terms of a severe hyposmic/anosmic group. Those candies being identified easiest by normosmics, but hardest by the second group were considered for the new 7-item CST. Eventually the test consisted of orange, anise, raspberry, vanilla, pineapple, coffee and coconut. Examinees had to choose one flavour out of the seven (given verbal and visual cues) in a non-forced-choice manner (providing "no flavour" and "undefinable" as an answer option). Tested subjects were randomly assigned to group $A(n=85)$ or $B(n=78)$ differing in administration order of the candies. Before enjoying the candies, participants had to read the following instructions: "For this test you have to put the candy in your mouth and suck on it (without smelling it first). Please then fill in the number of the candy in the provided field next to the picture/word best fitting to perceived flavour. If you do not experience any flavour or are unable to define the flavour, please fill in appropriately. Each number has to be filled in somewhere. You may also correct your answers." In a subgroup of 30 subjects (19 healthy participants and 11 OD patients) we performed a second testing cycle a minimum of three days after the first testing cycle (19.6 \pm 21.3 days).

\section{Cognitive tests and Questionnaires}

Cognitive tests and questionnaires were performed in between ortho- and retronasal tests providing "olfactory breaks". In all adult participants of study II and those performing testretesting in study l, the Mini-mental-state-examination (MMSE) was obtained, a short test of overall cognitive function with a maximum score of 30 and a cut-off of 24 for normal cognitive function (26).

All participants rated their abilities to smell (subjective assessment of smell, SAS) and perceive detailed flavours (subjective assessment of flavour, SAF) like wine and herbs on a ten-point scale (1=no smell/flavour, 10=excellent smell/flavour perception) before psychophysical testing.

\section{Statistical analysis}

IBM SPSS 23.0 (IBM Corp., Armonk, NY, USA) and R version 3.5.1 (R Foundation for Statistical Computing, Vienna, Austria) with the R-libraries $P R O C{ }^{(27)}$ were used for statistical analysis. Same $R$ version was used to visualize data. In case of Figure 2 and 3 GraphPrism 7.0 (GraphPad Software, La Jolla, CA, USA) was used for visualization. To compare scores in an explorative manner, we used ANOVA and unpaired two-sample t-tests without adjustment for multiple comparisons. Correlational analyses were performed using the Pearson correlation coefficient ( $r$ ). The alpha level was set at 0.05 . For test-retest reliability the intraclass correlation coefficient (ICC) was calculated, in case of the single candies of the 7-item CST Cohen's kappa ( $\kappa$ ) was used. The diagnostic power was analysed with receiver operating characteristic curves (ROC). CST score results for healthy, hyposmic and anosmic subjects were visualized with boxplots and presented in a table. Presented descriptive statistics for all metric values are Mean $\pm S D$, relative and absolute frequencies are shown for categorical variables.

The number of candies in the short version was set to 7 a priori by comparing the distributions of score results in case of sheer guessing among different CST test sizes: The probability to have 4 correct answers by sheer guessing is only $1.83 \%$ with seven flavours, not taking into account the answer options "no flavour" and "undefinable". Although this percentage decreases when adding more possible answers (in case of seven candies and eight answers: $1,1 \%$; in case of nine answers: $0.7 \%$ ), investigators concluded a priori this would not increase test accuracy (by highly increasing test difficulty to healthy subjects). Sample size for study II was set to $n=50$ patients and $n=100$ healthy subjects expecting to find a cut-off with at least 0.8 sensitivity and 0.8 specificity, in which case the width of 95\% confidence interval for the sensitivity would be smaller or equal 0.088 and for the specificity smaller or equal 0.062 .

\section{Results}

Study I

CST scores of hyposmic subjects $(n=36,13.5 \pm 4.7)$ differed significantly from normosmics $(n=49,19.7 \pm 3.4)(p<0.0001)$, as well as 
Table 1. Correct answers on all 27 candy aromas.

\begin{tabular}{|c|c|c|c|c|c|c|c|}
\hline Aroma & $\begin{array}{c}(\mathrm{N}=49) \\
\text { Normosmia }\end{array}$ & $\begin{array}{l}\text { Hit rate } \\
\begin{array}{l}(\mathrm{N}=\mathbf{3 4}) \\
\mathrm{TDI} \leq 20\end{array}\end{array}$ & Diff. & Aroma & $\begin{array}{c}(\mathrm{N}=49) \\
\text { Normosmia }\end{array}$ & $\begin{array}{l}\text { Hit rate } \\
\begin{array}{l}(\mathrm{N}=\mathbf{3 4}) \\
\mathrm{TDI} \leq \mathbf{2 0}\end{array}\end{array}$ & Diff. \\
\hline Anise & 1.000 & 0.088 & 0.912 & Cinnamon & 0.735 & 0.353 & 0.382 \\
\hline Pineapple & 0.878 & 0.118 & 0.760 & Gingerbread & 0.735 & 0.382 & 0.352 \\
\hline Coffee & 0.959 & 0.206 & 0.753 & Blackcurrant & 0.714 & 0.382 & 0.332 \\
\hline Coconut & 0.918 & 0.265 & 0.654 & Apricot & 0.755 & 0.441 & 0.314 \\
\hline Orange & 0.857 & 0.206 & 0.651 & Condens.Milk & 0.837 & 0.529 & 0.307 \\
\hline Lemon & 0.857 & 0.265 & 0.592 & Waldmeister & 0.510 & 0.206 & 0.304 \\
\hline Rhubarb & 0.776 & 0.206 & 0.570 & Eisbonbon & 0.612 & 0.324 & 0.289 \\
\hline Peach & 0.837 & 0.294 & 0.543 & Nut-Nougat & 0.571 & 0.294 & 0.277 \\
\hline Vanilla & 0.816 & 0.294 & 0.522 & Plum & 0.510 & 0.235 & 0.275 \\
\hline Raspberry & 0.837 & 0.382 & 0.454 & Banana & 0.612 & 0.441 & 0.171 \\
\hline Mandarin & 0.714 & 0.265 & 0.450 & Hazelnut & 0.449 & 0.294 & 0.155 \\
\hline Cola & 0.653 & 0.206 & 0.447 & Peppermint & 0.939 & 0.853 & 0.086 \\
\hline Cherry & 0.633 & 0.206 & 0.427 & Apple & 0.306 & 0.235 & 0.071 \\
\hline Pear & 0.694 & 0.294 & 0.400 & & & & \\
\hline
\end{tabular}

Participants scoring equal or above 30.75 on TDI were referred to as normosmia group $(n=49)$ and those scoring equal or below 20 on TDI in terms of a severe hyposmia / anosmia group $(n=34)$. Diff.: indicating differences of hit rates amongst the two groups, ordered from highest to lowest differences (for instance, anise was named correct by $100 \%$ of the healthy subjects, whereas only $8.8 \%$ of the subjects scoring 20 or less on TDI were able to identify anise.) TDI, summed score of orthonasal odor threshold, discrimination and identification testing; Aromas in bold were chosen for the new 7-item Candy Smell Test.

CST scores of anosmics $(n=26,7.6 \pm 2.8)$ from hyposmics scores $(p$ $<0.0001$ ). None of the anosmic participants scored above 13 on the CST, the proposed cut-off for anosmia for the initial 23-CST ${ }^{(16,24)}$. Hit rates of all aromas are illustrated in Table 1. ROF (27CST) correlated significantly with orthonasal olfactory function (OOF), as measured by $\mathrm{T}(\mathrm{r} 111=0.618, \mathrm{p}<0.0001), \mathrm{D}(\mathrm{r} 111=$ $0.730, \mathrm{p}<0.0001), \mathrm{I}(\mathrm{r} 111=0.834, \mathrm{p}<0.0001)$, and the summed TDI ( $r 111=0.833, \mathrm{p}<0.0001)$.

For home-testing, mean time between the test cycles was $63.0 \pm 49.2$ days. One participant reported 2 candies were split into a few pieces after mailing, nevertheless testing was possible. Two CST answer sheets (not included in 25 subjects) were not returned or lost on the mail route. Provided instructions on the candy chains also included a reminder on the forced-choice principle and in consequence no answer lines were skipped. Overall test-retest reliability was highly significant (ICC54 = $0.876, \mathrm{p}<0.0001$ ), aligning with previous findings ${ }^{(16)}$. Testretesting for home-testing correlated highly significant (ICC25 $=0.870, p<0.0001$ ) (Figure 2), whereas test-retest with both sessions at the clinic showed a similar correlation $($ ICC29 $=0.813$, $\mathrm{p}<0.0001)$.

\section{Study II}

Included normosmic subjects scored $13.7 \pm 1.2$ on orthonasal identification testing and $6.0 \pm 1.3$ on the new 7-item CST. OD patients scored $17.7 \pm 6.5$ on TDI testing and $1.8 \pm 1.8$ on the new 7-CST. There was no significant difference in testing performance in regard to test sequence (Group A and B; $p>0.268$; Mantel-Haenszel-test). Scores on the 7-CST significantly correlated with OOF as measured by TDI testing in OD patients ( $\mathrm{r} 47$ $=0.669, p<0.0001$ ) and weakly in healthy subjects as measured by identification testing $(r 116=0.218, p=0.0185)$. Figure 3 illustrates 7-CST results for healthy participants, hyp- and anosmics. None of the anosmic subjects were able to obtain a score of more than three on the 7-CST, whereas only eight healthy subjects scored less than four. The full 27-CST may take beyond 20 minutes to test, the 7-CST only took between 5 to 10 minutes. The 7-CST score showed high diagnostic capacities to identify OD patients in general (ROC area under the curve (AUC): 0.9485 (Figure 4) and anosmic patients (ROC AUC: 0.9788). For ROC coordinates and Youden's Index at defined cut-offs see Table 2. In the subgroup of 30 subjects the 7-CST score was $4.4 \pm 2.6$ for the first session and $4.6 \pm 2.7$ for the second session. Healthy participants tended to perform better the second time (5.8 \pm 1.7 vs. $6.4 \pm 1.1)$. Test-retesting showed a significant correlation (ICC30 = $0.759, \mathrm{p}<0.0001)$. Looking at each aroma separately, test-retesting revealed substantial agreement for pineapple $(\kappa=0.791, p$ $<0.0001)$, anise $(\kappa=0.718, p<0.0001)$ and orange $(\kappa=0.615, p$ 


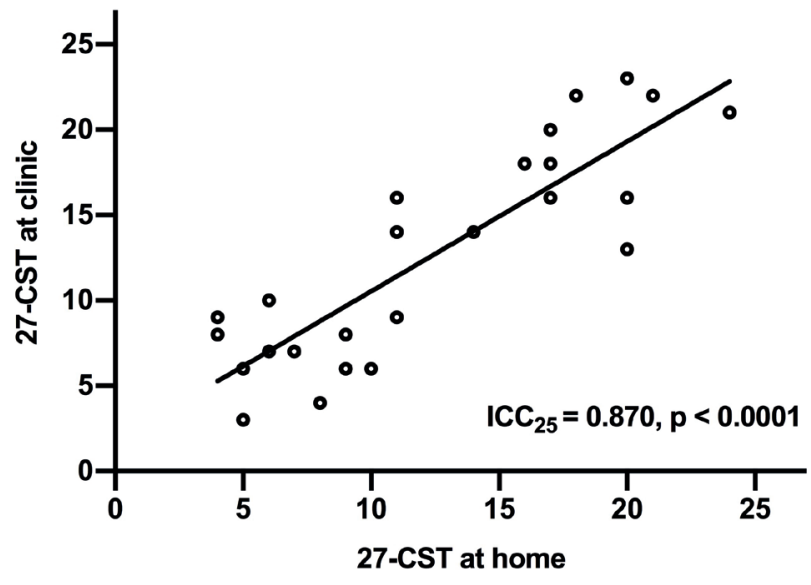

Figure 2. Linear regression graph of participants performing one test cycle at the clinic and one at home being supplied with illustrated candy chains. The intraclass correlation coefficient (ICC) showed a strong correlation.

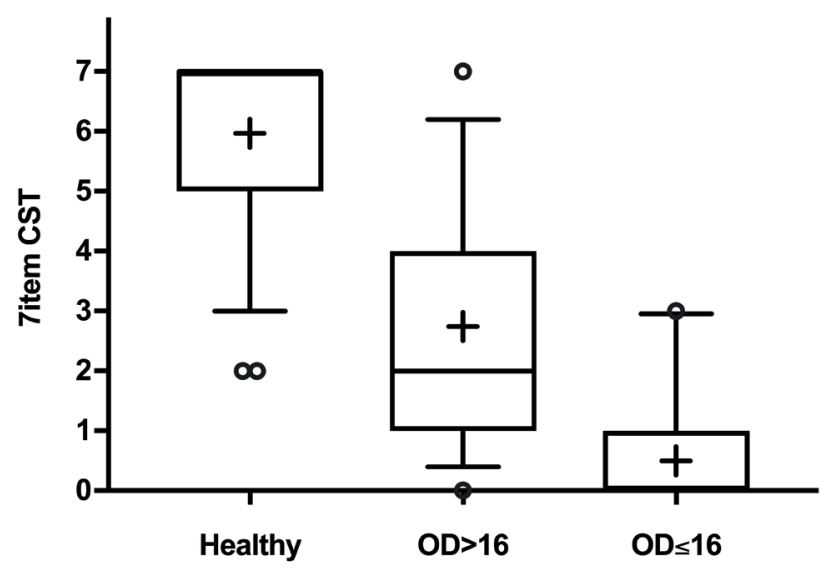

Figure 3. Box-and-whisker plots of the new 7-item Candy Smell Test. Scores in healthy, hyposmic (> 16 and below 30.75 TDI) and anosmic ( $\leq$ $16 \mathrm{TDI})$ subjects. Medians (Q0.5; line), interquartile range (Q0.25, Q0.75; boxes); + indicating the mean scores; OD.: olfactory dysfunction; Outlier: one anosmic subject scored 3 points. None of the subjectively healthy subjects scored less than 2 points.

$=0.0007)$. Moderate agreement was found for coffee $(\kappa=0.556$, $\mathrm{p}=0.0022$ ). Test-retesting did not show relevant agreement for coconut, vanilla and raspberry (all $\kappa<0.349$, all $p>0.05$ ).

Concerning the orthonasal 16-identification test, healthy subjects were able to identify turpentine only in $36 \%$ and apple in $54 \%$ correctly, followed by lemon (74\%), pineapple (76\%) and liquorice (86\%). All other odors were identified in more than $91 \%$ of the cases. Only 2 subjectively healthy subjects scored at the 10th percentile in identification testing. In tested children/adolescents only one subject scored one point below the 10th percentile of the orthonasal Sniffin ' kids test ${ }^{(25)}$. On the new 7-CST this small group scored rather low

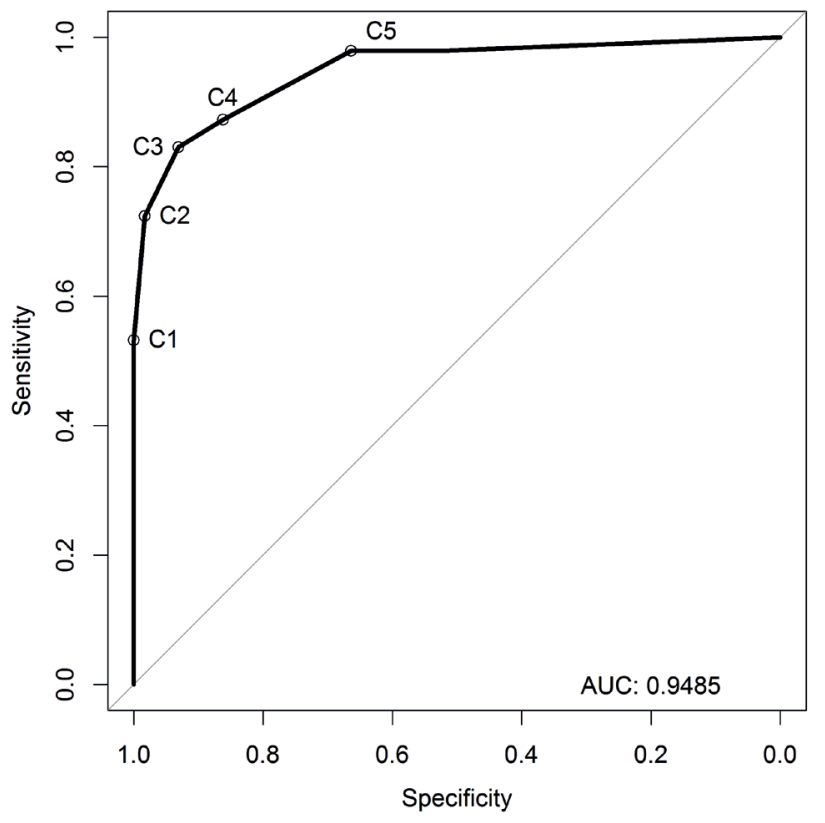

Figure 4. Receiver operating characteristic curve of the new 7-item Candy Smell Test. The area under the curve (AUC) indicates high diagnostic capacities to differentiate healthy subjects from patients with olfactory dysfunction. $\mathrm{C} 1=$ Cut-off $\leq 1, \mathrm{C} 2=$ Cut-off $\leq 2, \mathrm{C} 3=$ Cut-off $\leq 3$, C4=Cut-off $\leq 4, C 5=$ Cut-off $\leq 5$.

\section{(3.5 \pm 1.9$)$.}

\section{Questionnaires}

The overall mean MMSE score was $29.9 \pm 0.6$ and no subject scored below 24, indicating normal cognitive function. In overall healthy subjects SAS and SAF did not correlate significantly with orthonasal nor with retronasal odor identification abilities $(p>$ $0.05)$. In overall subjects scoring below the normative range of normosmia SAS correlated significantly with TDI $\left(r_{109}=0.655\right.$, $p<0.0001)$ and its subscales $(p<0.05)$. SAF weakly correlated with T, I and TDI (all $r_{109}<0.269, p<0.05$ ). ROF in OD patients correlated significantly with SAS for the 27-CST $\left(r_{62}=0.514, p<\right.$ $0.0001)$ and the 7-CST $\left(r_{47}=0.491, p<0.0001\right)$ but not with SAF $(p>0.05)$.

\section{Discussion}

In contrast to a very large number of orthonasal olfactory tests ${ }^{(14,28)}$, considerably fewer publications focus on retronasal olfactory testing in a clinical aspect ${ }^{(12)}$. A high clinical and research interest for retronasal olfaction however is evident (e.g., rhinologic research and questions on nutrition and food preferences), promoting availability and further standardization of testing methods is necessary. In this investigation, we propose a suitable strategy (using candy-chains) for postal distribution of candies, home testing of ROF and we found highly comparable retronasal test results in an unmonitored remote home-setting. 
Table 2. Coordinates of the receiver operating characteristic curve, Cutoffs and Youden's Index of the new 7-item Candy Smell Test.

\begin{tabular}{|cccc|}
\hline Specificity & Sensitivity & Cut-off & Youden's Index \\
\hline 0.98 & 0.53 & $\leq 6$ & 0.51 \\
0.98 & 0.66 & $\leq 5$ & 0.64 \\
0.87 & 0.86 & $\leq 4$ & 0.73 \\
0.83 & 0.93 & $\leq 3$ & 0.76 \\
0.72 & 0.98 & $\leq 2$ & 0.71 \\
0.53 & 1.00 & $\leq 1$ & 0.53 \\
0.30 & 1.00 & $=0$ & 0.30 \\
\hline
\end{tabular}

When defining scores of $\leq 2$ as diseased, the test has a specificity of 0.72 and sensitivity of 0.98 . According to the Youden 's Index a score $\leq 3$ would be ideal for distinguishing healthy subjects from diseased. Given a larger variance in scores in included hyposmic (see also Fig. 2), further olfactory testing is recommended for scores $\leq 4$.

Furthermore, we advocate a new retronasal screening strategy: The 7-CST showed to be highly capable of distinguishing between norm- and anosmic subjects.

Notable advantages of applied candies are e.g., fairly stable aroma concentrations, potential for self-administration, high acceptance in children ${ }^{(16)}$, close to colourlessness (no visual bias) and an adequate shelf-life. However, shelf-life limits need further evaluation (candy-chains were all spent within five months).

This application of the 27-CST produced similar results to former studies ${ }^{(16,24)}$. Novel items were recognized at expectable rates.

Larger populations are needed to establish normative data and cross-cultural validation is certainly pending.

Presented candy-chains for self-testing and postal distribution are a modified packing method for daily medications. Illustrated packing is intended to reduce chances of confusion of morning, midday and evening pills and save time. In regard to the CST, packing was suitable for forming candy-chains of 27 bags, but also seven bags for the new screening test are conceivable and need validation. The scratch-and-sniff technique (i.e., UPSIT) seems to be the most common strategy for post-delivery of olfactory tests. Disadvantages (e.g., failure of odorants to encapsulate) have been addressed and postal distribution of tests in general has its pitfalls as well (e.g., low response rates if randomly mailed) (29). A potential advantage of retronasal testing in a home setting: subjects may not be tempted to seek assistance in flavour selection, whereas in orthonasal home testing also e.g., nearby family members may influence answers. In this investigation participation compliance was high, which explains that only two answer sheets were not returned.

In orthonasal olfactory testing, screening tests usually rely on more than seven stimuli (e.g., 12 stimuli for the 12-item Sniffin' Sticks test ${ }^{(10)}$ and also 12 for the popular scratch-and-sniff test
B-SIT (9)) and commonly supply four possible answers for one stimulus in a forced-choice paradigm. This strategy allows high scores by sheer guessing. Reducing stimuli, but increasing number of descriptors can overcome this issue, as proposed in a short 5 -item Sniffin 'Sticks screening test ${ }^{(11)}$. Also, since the 7-CST was not intended to uncover malingering subjects (as most screening tests are not capable of) we decided to apply seven cues for seven candies and provide "undefinable" and "no flavour" as answer options. Notable, OD patients were very "conservative" or less gambling in regard to the 7-CST test setting: rather than guessing, the alternative answers were appointed very frequently (out of 47 OD patients "no flavour" was selected at least once in 17 and "undefinable" at least once in 28 tests). Patient honesty on flavour perception therefore may have contributed to presented results and underlines that paradigm selection influences test results in psychophysical testing, as it has recently been shown for gustatory testing ${ }^{(30)}$.

Given the present findings on the new 7-CST, we suggest further olfactory tests should be advised in subjects scoring less than five points, with a high probability of subjects being anosmic or severely hyposmic when scoring zero or one on the 7-item CST. Scores of equal or more than five most likely represent normal olfactory function or slight hyposmia. In regard to a shorter screening test for children further studies are needed, supposedly with a less complex structure.

The final candy selection of the 7-CST was not strictly according to best performing candies (Table 1). Investigators found lemon and peach too similar in flavour. Rhubarb was found to potentially put a cultural bias on the test a priori. Interestingly, the alternatively selected candies (vanilla and raspberry) performed worst in test-retesting, which highlights the need for further studies on different flavour combinations and also validation in different cultural settings with larger cohorts.

In clinical routine smell (and flavour) complains are often communicated in "food terms". In return, estimations exist that the main part of what we consider "food taste" is in fact due to odour perception (31). Even if the term "flavour" is not used appropriately, the combination of "taste and smell" complaints predominantly indicate smell impairment rather than gustatory dysfunction when tested ${ }^{(32)}$. Interestingly, included OD patients were able to "foresee" their orthonasal olfactory abilities by a subjective rating quite precisely (aligning with previous findings $\left.{ }^{(33-35)}\right)$, but flavour perception ratings did not reflect retronasal test results. These circumstances justify retronasal smell tests beyond mentioned "educational" benefit.

Another reason supporting the application of retronasal tests in regard to a rhinologic work-up: Obstruction of the olfactory cleft can lead to differences in OOF and ROF ${ }^{(7,36)}$. These differences may lack if not nasal polyps (obstruction), but olfactory epithelium specific diseases (i.e., long lasting inflammation) cause dysfunction. The usage of ortho- and retronasal olfactory 
tests together may therefore uncover obstructive processes (in or neighbouring the olfactory cleft) and hence possibly serve as follow-up tools in CRSwNP in future - potentially delivered by post.

\section{Conclusion}

The present investigation shows the Candy-Smell-Test to be suitable for home-testing of retronasal olfactory function with the possibility of postal distribution. The new 7-item CST enables a rapid evaluation of retronasal function and unmasks anosmic subjects. These findings help in further standardizing retronasal olfactory tests, may encourage rhinologists to more routinely evaluate retronasal olfactory abilities and pave the way for larger epidemiologic studies also in regard to food preferences and nutritional behaviour.

\section{Acknowledgements}

This study obtained no third-party funding. The authors greatly thank Dr. E. Salamon and the "Marien Apotheke, Wien" for professionally packing candies on a goodwill basis. Study I was granted the Heinz-Kurz-Poster-Price (2nd place) namely to G. Besser at the 62nd annual congress of the Austrian ENT Society 2018 (Bregenz). Study II was presented at the 90th annual congress of the German ENT Society 2019 (Berlin). J. Waldmann collected data in children. We thank Dr. S. Seyferth for professionally manufacturing candies and Frey\&Lau GmbH for providing aromas.

\section{Authorship contribution}

GB: concept of study, collection of data, analysis of results, writeup of manuscript, critical review of all contents; DTL, BP and DI: collection of data, critical review of all contents; EP: concept of study (statistics), analysis of results; CAM: concept of study, critical review of all contents; BR: concept of study, critical review of all contents.

\section{Conflict of interest}

All authors state that they have no conflicts of interest.

\section{References}

1. Hummel T, Seo H-S. Chapter 13 - Orthonasa and retronasal perception. In: Guichard E, Salles C, Morzel M, Le Bon A-M, eds. FLAVOUR - From Food to Perception. Chichester, West Sussex: Wiley Blackwell, 2017; 310-8.

2. Rozin P. "Taste-smell confusions" and the duality of the olfactory sense. Percept Psychophys 1982; 3:397-401.

3. Murphy C, Cain WS. Taste and olfaction: independence vs interaction. Physiol Behav 1980; 24:601-5.

4. Small DM, Gerber JC, Mak YE, Hummel T. Differential neural responses evoked by orthonasal versus retronasal odorant perception in humans. Neuron 2005; 47:593605.

5. Blankenship ML, Grigorova M, Katz DB Maier JX. Retronasal Odor Perception Requires Taste Cortex, but Orthonasal Does Not. Curr Biol 2019; 29:62-3.

6. Pierce AM, Simons CT. Olfactory Adaptation is Dependent on Route of Delivery. Chem Senses 2018; 43:197-203.

7. Landis BN, Giger R, Ricchetti A, et al Retronasal olfactory function in nasal polyposis. Laryngoscope 2003; 113:1993-7.

8. Othieno F, Schlosser RJ, Storck KA, Rowan NR, Smith TL, Soler ZM. Retronasal olfaction in chronic rhinosinusitis. Laryngoscope 2018; 128:2437-42

9. Doty RL, Marcus A, Lee WW. Development of the 12-item Cross-Cultural Smell Identification Test (CC-SIT). Laryngoscope 1996; 106:353-6.

10. Hummel $T$, Konnerth $C G$, Rosenheim $K$ Kobal G. Screening of olfactory function with a four-minute odor identification test: reliability, normative data, and investiga- tions in patients with olfactory loss. Ann Otol Rhinol Laryngol 2001; 110:976-81.

11. Mueller C, Renner B. A new procedure for the short screening of olfactory function using five items from the "Sniffin' Sticks" identification test kit. Am J Rhinol 2006; 20:113-6

12. Rimmer J, Fokkens WJ, Hellings $P$, et al. European Position Paper on Diagnostic Tools in Rhinology. Rhinol Suppl 2019; 28:142.

13. Doty RL, Shaman P, Dann M. Development of the University of Pennsylvania Smell Identification Test: a standardized microencapsulated test of olfactory function. Physiol Behav 1984; 32:489-502.

14. Doty RL. Office procedures for quantitative assessment of olfactory function. Am J Rhinol 2007; 21:460-73.

15. Heilmann S, Strehle G, Rosenheim K, Damm $M$, Hummel T. Clinical assessment of retronasal olfactory function. Arch Otolaryngol Head Neck Surg 2002;128:414-8.

16. Renner B, Mueller CA, Dreier J, Faulhaber S, Rascher W, Kobal G. The candy smell test: a new test for retronasal olfactory performance. Laryngoscope 2009; 119:487-95.

17. Maione L, Cantone E, Nettore IC, et al. Flavor perception test: evaluation in patients with Kallmann syndrome. Endocrine 2016; 52:236-43.

18. Pal P, Shepherd D, Hamid N, Hautus MJ. The use of freeze-dried retronasal stimuli to assess olfactory function. Clin Otolaryngol 2019; coa.13389.

19. Kobal G, Hummel T, Sekinger B, Barz S, Roscher S, Wolf S. "Sniffin' sticks": screening of olfactory performance. Rhinology 1996; 34:222-6.

20. Hummel T, Sekinger B, Wolf SR, Pauli E
Kobal G. "Sniffin" Sticks': olfactory performance assessed by the combined testing of odor identification, odor discrimination and olfactory threshold. Chem Senses 1997; 22:39-52.

21. Hummel T, Kobal G, Gudziol H, Mackay-Sim A. Normative data for the "Sniffin' Sticks" including tests of odor identification, odor discrimination, and olfactory thresholds: an upgrade based on a group of more than 3,000 subjects. Eur Arch Otorhinolaryngol 2007; 264:237-43.

22. Oleszkiewicz A, Schriever VA, Croy I, Hähner A, Hummel T. Updated Sniffin' Sticks normative data based on an extended sample of 9139 subjects. Eur Arch Otorhinolaryngol 2018; 34:222-10.

23. Liu DT, Besser G, Renner B, Seyferth $S$ Hummel T, Mueller CA. Retronasal olfactory function in patients with smell loss but subjectively normal flavor perception. Laryngoscope 2019;57:639.

24. Haxel BR, Bertz-Duffy S, Faldum A, et al. The Candy Smell Test in clinical routine. Am J Rhinol Allergy 2011;25:145-8.

25. Schriever VA, Mori E, Petters W, Boerner C. Smitka M, Hummel T. The "Sniffin' Kids" Test - A 14-Item Odor Identification Test for Children. PLoS One 2014; 9:e101086.

26. Folstein MF, Folstein SE, McHugh PR. "Minimental state." J Psychiatr Res 1975; 12:189_ 98.

27. Robin X, Turck N, Hainard A, et al. pROC: an open-source package for $\mathrm{R}$ and $\mathrm{S}+$ to analyze and compare ROC curves. BMC Bioinformatics 2011; 12:77

28. Hummel $T$, Whitcroft $K$, Andrews $P$, et al. Position paper on olfactory dysfunction. Rhinol Suppl 2017; 54:1-30.

29. Snitz K, Perl O, Honigstein D, et al. 
SmellSpace: An Odor-Based Social Network as a Platform for Collecting Olfactory Perceptual Data. Chem Senses 2019; 44:267-78.

30. Besser G, Prassl A, Mueller CA, Renner B. Testing gustatory function using either a forced-choice or a non-forced-choice paradigm - Does it make a difference? Rhinology 2019; 57:385-391

31. Breer H. The sense of smell: reception of flavors. Ann N Y Acad Sci 2008; 1126:1-6.

32. Hunt JD, Reiter ER, Costanzo RM. Etiology of subjective taste loss. Int Forum Allergy Rhinol 2019; 9:409-12.

33. Kollndorfer K, Kowalczyk K, Nell S, Krajnik J, Mueller CA, Schöpf V. The inability to self-evaluate smell performance. How the vividness of mental images outweighs awareness of olfactory performance. Front Psychol 2015; 6:627.

34. Besser G, Liu DT, Renner B, Hummel T, Mueller CA. Olfactory implant: Demand for a future treatment option in patients with olfactory dysfunction. Laryngoscope 2018 127:497.

35. Lötsch J, Hummel T. Clinical usefulness of self-rated olfactory performance - a data science-based assessment of 6000 patients. Chem Senses 2019; 44:357-364.

36. Pfaar $\mathrm{O}$, Landis BN, Frasnelli J, Hüttenbrink K-B, Hummel T. Mechanical obstruction of the olfactory cleft reveals differences between orthonasal and retronasal olfactory functions. Chem Senses 2006; 31:27-31.
Christian A. Mueller, MD

Department of Otorhinolaryngology

and Head and Neck Surgery

Medical University of Vienna

Währinger Gürtel 18-20

1090 Vienna

Austria

Tel: +4314040033340

E-Mail:

christian.a.mueller

@meduniwien.ac.at 